\title{
Strumaprophylaxe - wann endlich?
}

Krankheitsverhütung wurde von der gegenwärtigen Bundesregierung wiederholt als vordringlich propagiert. Wcr die Passivität auf dem Sektor Strumaprophylaxe rund 10 Jahre lang verfolgt hat, beginnt allmählich an der Aufrichtigkeit dieser Vorsätze zu zweifeln. Es ist nicht zu verstehen, warum die Möglichkeit von unseren Politikern nicht wahrgenommen wird, mit einfachen und übersichtlichen Maßnahmen, die noch dazu kaum nennenswerte Kosten verursachen, die Strumahäufigkeit in der Bevölkerung der Bundesrepublik von jetzt mindestens $15 \%$ auf höchstens $3 \%$ zu senken. Wahrscheinlich gibt es kein lohnenderes Beispiel für die Prophylaxe überhaupt. Auf jeden Fall ist ein "Kostendämpfer" suspekt, der sich ausgerechnet bei diesem eklatanten Fall inaktiv verhält. Bekanntlich spielt die Kostenfrage im Gesundheitswesen der Entwicklungsländer eine noch entscheidendere Rolle als in der Bundesrepublik; hier könnten wir von den Entwicklungsländern lernen, denn sic haben es in größerer Zahl verstanden, mit der Strumaprophylaxe letztlich äußerst wirksam Krankheitskosten zu sparen.

Unser Lebensmittelrecht verbietet Zusätze zu Nahrungsmitteln, auf dic jeder Mensch angewiesen ist. Die zur Strumaprophylaxe geeigneten jodierten Speisesalze müssen daher als diätetische Lebensmittel auf freiwilliger Basis benutzt werden. Die Sektion Schilddrüse der Deutschen Gesellschaft für Endokrinologie ist von diesem Freiwilligkeitsprinzip deswegen nicht sehr begeistert, weil sie fürchtet, daß man nur mit großen Schwierigkeiten die Bevölkerung in der notwendigen Vollständigkeit aufklären kann. Es ist auch unlogisch, einerseits die Trinkwasserchlorierung aus hygienischen Gründen zuzulassen und andererseits die generelle Zugabe von Jod zum Speisesalz als unethisch zu bezeichnen. Der Jodmangel ist ein Problem der mangelhaften Ernährung, und seine Beseitigung ist eine hygienische Maßnahme. In diesem Sinne ist nicht-jodiertes Speisesalz unhygienisch.

Weil nun für die nächsten Jahre das Freiwilligkeitsprinzip bei der Benutzung von jodiertem Speisesalz beibehalten werden soll, müssen alle Beteiligten, auch unserc Regicrung, das ihre tun, um diese Form der

Dtsch. med. Wschr. 107 (1982), 843-844

(c) 1982 Georg Thieme Verlag Stuttgart - New York

0012-0472/82 0604-0843 \$02.00
Strumaprophylaxe zu dem gewünschten Erfolg zu führen. Jedermann muß wissen, daß unsere Ernährung jodarm ist und daß dieser Jodmangel ausgeglichen werden muß. Jodierte Speisesalze im Sinne der Diätverordnung (6. Verordnung zur Änderung der Diätverordnung vom 7 . Juli 1981, Bundesgesetzblatt, Jahrgang 1981, Teil I, Scitc 613, Paragraph 3, Paragraph 10 Absatz 1, 2 und 4 usw.) sind regional als Düra-Vollsalz und als Bayerisches Vollsalz, bundesweit als Bad Reichenhaller Jodsalz überall im Handel erhältlich.

Da die jüngste Änderung der Diätverordnung uns jetzt endlich ein jodiertes Speisesalz beschert hat, das sich zur Strumaprophylaxe vollauf eignet, kommt es nunmehr darauf an, die Bevölkerung vollständig und kontinuierlich aufzuklären. Deshalb wird gefordert:

1. Die Kultusministerkonferenz der Länder möge beschließen, daß die Lehrpläne sämtlicher Schulen obligat den gesamten Themenbereich "Jodmangel in der Nahrung - Anpassung der Schilddrüse durch Vergrößerung - Kropfleiden - Verhütung desselben durch jodiertes Speisesalz " enthalten. Das ist ein praktisch wichtiges, didaktisch gut geeignetes Bcispiel für den Biologieunterricht.

2. Die Kropfhäufigkeit muß bei allen Schulkindern durch die schulärztliche Untersuchung in regelmäßigen Abständen dokumentiert werden. Bei dieser Grelegenheit muß allen Schulkindern ein Aufklärungsblatt über die Notwendigkeit der Strumaprophylaxe durch jodiertes Speisesalz ausgehändigt werden. Eine schriftliche Empfangsbestätigung durch die Erziehungsberechtigten wäre wünschenswert.

3. Besonders wirksam wären 30-60 Sekunden dauernde Fernsehspots unmittelbar vor den Abendnachrichten, mit denen man über die Notwendigkeit informieren könnte, das richtige $=$ jodierte Speisesalz zu benutzen. Solchc Fernsehspots sind für die Diabetes-Früherkennung ja schon gesendet worden.

4. Die Ernährungs- und Verbraucherberatung sollte sich ebenso wie die Krankenkassen dieses Themas annehmen.

5. Bei der Schwangerenberatung sollte die Empfehlung von jodiertem Speisesalz obligatorischer Bestandteil werden, da hier eine besonders lohnende Zielgruppe angesprochen würde. 
6. Darüber hinaus ist jeder einzelne Leser dieser Wochenschrift aufgerufen, diese Aufklärungsaktion nach besten Kräften zu unterstützen.

Man wird in Laufe der nächsten 2 bis 3 Jahre mit Sicherheit beginnen, wissenschaftliche Untersuchungen durchzuführen, um die Wirksamkeit der Strumaprophylaxe durch jodiertes Speisesalz auf freiwilliger Basis zu prüfen. Neben der Beurteilung der Häufigkeit der Schilddrüsenvergrößerung bei Schulkindern stehen hierfür die Beurteilung der alimentären Jodversorgung (Messung der Urin-Jodausscheidung) und der Vergleich der Absatzzahlen der Hersteller für jodiertes Speisesalz zur Verfügung.

Nur wenn es gelingt, die für die Vorbeugung besonders geeignete Gruppc der unter Vierzigjährigen vollständig zur Benutzung des jodierten Speisesalzes zu bringen und sie auch dabei zu halten, ist mit einer entscheidenden Verminderung der Strumahäufigkeit zu rechnen. Um der Klarheit willen: Sollten in einer Familie neben jüngeren Menschen auch über Vierzigjährige gemeinsam essen, so können alle Familienmitglieder zusammen ohne wesentliches Risiko das jodierte Speisesalz benutzen.

Fazit: Wir haben jetzt das richtige Salz, sorgen wir dafür, daß es praktisch ausschließlich benutzt wird.

Prof. Dr. P. C. Scriba

Sprecher der Sektion Schilddrüse der

Deutschen Gesellschaft für Endokrinologie

Klinik für Innere Medizin der

Medizinischen Hochschule

2400 Lübeck 1, Ratzeburgcr Allee 160 